\title{
Malformaciones congénitas en hijos de madres epilépticas con y sin tratamiento con anticonvulsivantes
}

\author{
Jazmín Arteaga-Vázquez, MC,(I) Leonora Luna-Muñoz, TS,(I) Osvaldo M Mutchinick, DC.(I)
}

\begin{abstract}
Arteaga-Vázquez J, Luna- Muñoz L, Mutchinick OM. Malformaciones congénitas en hijos de madres epilépticas con y sin tratamiento con anticonvulsivantes. Salud Publica Mex 2012;54:579-586.
\end{abstract}

\begin{abstract}
Resumen
Objetivo. Determinar la frecuencia y tipo de malformaciones congénitas (MC) en hijos de madres epilépticas (HME) tratadas y no tratadas con anticonvulsivantes, la posible correlación anticonvulsivante/MC y la asociación con otras alteraciones del desarrollo. Material y métodos. Estudio multicéntrico de casos y controles en 166 recién nacidos vivos $\mathrm{HME}$ identificados en $2 \mathrm{I} 50 \mathrm{I}$ recién nacidos con MC y respectivos controles del Registro y Vigilancia Epidemiológica de Malformaciones Congénitas (RYVEMCE). Resultados. La frecuencia de MC en HME tratadas fue mayor, (48.3\%) que en HME no tratadas (28.3\%); (RM= 2.37 IC95\% I.08$5.40), p=0.03$. Las $M C$ más frecuentes fueron espina bífida, anomalías en reducción de miembros, labio/paladar hendido, microcefalia, anotia/microtia, hipospadias, paladar hendido, polidactilia, anoftalmia/microftalmia y onfalocele. No hubo diferencias entre uso de mono o politerapia. La difenilhidantoína, carbamazepina y ácido valproico fueron los anticonvulsivantes más utilizados. Conclusiones. Los resultados confirman la teratogenicidad propia de la epilepsia y el efecto sinérgico de ciertos anticonvulsivantes, lo que evidencia la necesidad de un apropiado control periconcepcional de esta enfermedad y su tratamiento.
\end{abstract}

Palabras clave: anomalías congénitas; anticonvulsivantes; epilepsia
Arteaga-Vázquez J, Luna- Muñoz L, Mutchinick OM.

Congenital malformations in the offspring of epileptic mothers with and without anticonvulsant treatment.

Salud Publica Mex 20I2;54:579-586.

\begin{abstract}
Objective. To determine the prevalence at birth and type of congenital malformations (CM) in newborns of epileptic mothers (NEM) treated and not treated with anticonvulsants, the correlation anticonvulsant/CM and other developmental disorders. Materials and methods. Multicenter casecontrol study, in 166 live births NEM diagnosed in 21 50 I newborns with $\mathrm{CM}$ and respective controls from the Registro y Vigilancia Epidemiológica de Malformaciones Congénitas (RYVEMCE). Results. The frequency of CM in NEM treated with anticonvulsants was higher (48.3\%) than in NEM of untreated mothers (28.3\%), (OR= 2.37 IC95\% I.08-5.40), $\mathrm{p}=0.03$. CMs most frequently found were: spina bifida, limb reduction defects, cleft lip palate, microcephaly, anotia/microtia, hypospadias, polydactyly, cleft palate, anophthalmia/ microphthalmia and omphalocele. No differences among monotherapy and polytherapy were observed. Diphenylhydantoin, carbamazepine and valproic acid were the most frequently anticonvulsants used. Conclusions. Our results show the teratogenicity of epilepsy by itself, the synergistic effect of some anticonvulsants, and the need of an appropriate periconceptional control of the disease and treatment.
\end{abstract}

Key words: congenital abnormalities; anticonvulsants; epilepsy

(I) Departamento de Genética, Instituto Nacional de Ciencias Médicas y Nutrición Salvador Zubirán. México DF, México.

Fecha de recibido: 27 de abril de 2012 - Fecha de aceptado: 19 de junio de 2012

Autor de correspondencia: D.C. Osvaldo M. Mutchinick. Departamento de Genética,

Instituto Nacional de Ciencias Médicas y Nutrición Salvador Zubirán. Vasco de Quiroga 15, Sección XVI. I4000 Tlalpan, México, DF. Correo electrónico: osvaldo@unam.mx 
$\mathrm{L}$ a Organización Mundial de la Salud define a la epilepsia como "una afección neurológica crónica, caracterizada por dos o más convulsiones no provocadas y atribuidas a la descarga eléctrica excesiva y repentina de un grupo de neuronas". ${ }^{1}$ La prevalencia de la enfermedad en general es de 4 a 6 por 1000 , y es más frecuente en mujeres menores de 45 años. ${ }^{1-3}$ En la población mexicana ésta es de 3.9 por 1000, (IC95\% 2.3-6.5). ${ }^{4}$ Se estima que una de cada 200 mujeres embarazadas padece epilepsia, ${ }^{5}$ y se incrementa la frecuencia de las crisis en $30 \%$ durante el primer trimestre de la gestación en una proporción considerable. ${ }^{6}$ Esta característica condiciona el uso de anticonvulsivantes, incluso a dosis altas durante la gestación, en la mayoría de las mujeres epilépticas.,

Los primeros fármacos antiepilépticos se utilizaron en el año 1850, ${ }^{9}$ pero más de un siglo después surgió la sospecha de su efecto teratogénico, ${ }^{10}$ al haberse observado una mayor frecuencia de malformaciones congénitas (MC) en los hijos de madres epilépticas (HME) tratadas con fenobarbital. Posteriormente, se asoció el uso de éste y otros fármacos con un mayor riesgo para labio y paladar hendido en la descendencia. ${ }^{11}$ Diversos estudios confirmaron el efecto teratogénico de los anticonvulsivantes, siendo las siguientes MC las reportadas con mayor frecuencia: defectos del tubo neural (DTN), microcefalia, craniosinostosis, paladar hendido, labio hendido con o sin paladar hendido (LH +/ - PH), cardiopatías congénitas, polidactilia, hernia diafragmática, e hipospadias. 6,8,12,13

En la actualidad se acepta que el riesgo para $\mathrm{MC}$ en HME expuestas a anticonvulsivantes durante los primeros meses del embarazo va de 6 a $9 \%$ comparado con el riesgo de 2 a $3 \%$ en la población general. ${ }^{13-16} \mathrm{Si}$ se consideran también las MC menores, la prevalencia al nacimiento aumenta a $16.9 \% .^{8}$ Se sabe que las crisis convulsivas en la madre epiléptica condicionan hipoxia embrionaria y algunos autores señalan que este factor pudiera tener un efecto teratogénico sobre el embrión y el feto, ${ }^{14}$ y que la epilepsia materna per se contribuye en cierta medida a aumentar el riesgo de $\mathrm{MC}$ en los HME.

Sin embargo, diversos autores postulan que la frecuencia de MC en la descendencia de madres epilépticas no tratadas no difiere de la frecuencia en madres control, $\left(R M=1.92\right.$, IC 95\% 0.92-4.00), ${ }^{17}$ con frecuencias de 3.5 y $4.2 \%$ en la descendencia de mujeres epilépticas no tratadas y tratadas durante la gestación, respectivamente. Diversos autores han comunicado una mayor frecuencia (6.0 al 16.8\%) de MC en los HME tratadas con politerapia. 7,18

Se ha sugerido como posible favorecedor del efecto teratogénico de los compuestos antiepilépticos la deficiencia de folatos por deficiente ingesta, absorción o metabolismo de los mismos. ${ }^{19}$ También se postula que alteraciones en la detoxificación celular por variantes moleculares de la enzima epóxido hidrolasa incrementaría el riesgo de toxicidad fetal, secundario a la formación de radicales libres producidos por el metabolismo de los anticonvulsivantes. ${ }^{6,20}$ Lo anterior podría estar relacionado con el documentado efecto dosis / respuesta de una mayor prevalencia al nacimiento de MC mayores inducidas por carbamazepina, lamotrigina y ácido valproico. ${ }^{7}$

La epilepsia materna se relaciona con complicaciones obstétricas y perinatales como hipoxia fetal, acidosis, hemorragia intracerebral in utero, aumento de la frecuencia de pérdidas fetales, desprendimiento de placenta, restricción del crecimiento intrauterino, parto pretérmino, bajo peso al nacimiento, y mayor mortalidad perinatal.6,14,21-23 La frecuencia del parto por cesárea en madres epilépticas $(20.6 \%)$ es mayor que en no epilépticas $(11.0 \%),(R M=1.93$; IC 95\% 1.32.8). ${ }^{21}$ Otra secuela en HME es un déficit cognitivo de grado variable, al parecer relacionado con el tipo de anticonvulsivante y dosis utilizadas. ${ }^{13}$ Esta alteración se caracteriza por retraso psicomotor y de desarrollo del lenguaje, hiperactividad y déficit de atención. ${ }^{24}$

Los objetivos del presente estudio fueron determinar en la descendencia de madres epilépticas la relación de la ingesta y tipo de anticonvulsivante con la frecuencia y tipo de MC y otras alteraciones del desarrollo como parto pretérmino, antecedente de abortos espontáneos y características somatométricas del recién nacido. Además considerar otros aspectos demográficos y reproductivos como edad materna, escolaridad de los padres y nivel socioeconómico como posibles factores de riesgo.

\section{Material y métodos}

La información se obtuvo de la base de datos del programa mexicano de "Registro y Vigilancia Epidemiológica de Malformaciones Congénitas" (RYVEMCE), coordinado en el Departamento de Genética del Instituto Nacional de Ciencias Médicas y Nutrición "Salvador Zubirán" (INCMNSZ). El RYVEMCE es un estudio multicéntrico con un diseño de casos y controles en recién nacidos vivos y muertos, basado en el examen clínico sistematizado de todos los nacimientos consecutivos que ocurren en los hospitales que participan del programa. Por cada recién nacido con malformaciones congénitas (RNMC) se incluye un recién nacido (RN) control sin $\mathrm{MC}$, que es el nacimiento siguiente al RNMC del mismo sexo nacido en el mismo hospital. De esta manera se tiene un conjunto de casos y controles pareados por sexo, lugar y momento del nacimiento. De cada RNMC y control se obtiene información uniforme por medio del 
llenado de una ficha clínica específicamente diseñada para el RYVEMCE. En ella se consignan datos clínicos del RN que incluyen peso, talla y perímetro cefálico, factores genéticos como antecedentes familiares de MC, consanguinidad y de exposición durante la gestación a factores químicos, físicos, infecciosos y de enfermedades maternas agudas y crónicas presentes durante la gestación. Del RNMC se describe detalladamente la o las MC presentes, y de éste y el RN control, información sobre la historia reproductiva de las respectivas madres e información sociodemográfica de las familias. ${ }^{16}$

El periodo de estudio comprendió de enero de 1978 a diciembre de 2010 y participaron 36 hospitales de diferentes estados de la República Mexicana. El protocolo de investigación fue revisado por el Comité de Bioética e Investigación en Humanos del INCMNSZ y cada hospital consideró su participación acorde con los lineamientos de su Comité de Bioética de Investigación en Humanos y criterios de obtención del consentimiento informado.

El diagnóstico de epilepsia, así como el de la ingesta de anticonvulsivantes, se obtuvieron por interrogatorio directo a la madre durante las primeras 24 horas después del parto o por consulta del expediente obstétrico, por lo que no fue posible obtener la dosis específica del anticonvulsivante utilizado.

Para el análisis estadístico de la información obtenida se utilizaron las pruebas de ji cuadrada de Pearson y exacta de Fisher para la comparación de las variables cualitativas entre casos y grupos de comparación, y la prueba " $\mathrm{t}$ " de Student para la comparación de las variables de distribución continua. La comparación de las prevalencias de las MC en los grupos de estudio se realizó mediante la prueba de distribución de Poisson (http:/ / stattrek.com/online-calculator/ poisson.aspx). Como valor de significancia estadística para todas las comparaciones realizadas se consideró una $\mathrm{p} \leq 0.05$.

\section{Resultados}

Durante el periodo señalado se examinaron un total de $1161510 \mathrm{RN}$, de los cuales 21501 correspondieron a $\mathrm{RN}$ vivos que presentaron una o más $\mathrm{MC}$ y un número igual de RN control. En el total de 43002 nacimientos se identificaron $174 \mathrm{RN}$ vivos con el antecedente de epilepsia materna, lo que da una prevalencia del 4.1 por 1000 de la enfermedad en mujeres en edad reproductiva (figura 1).

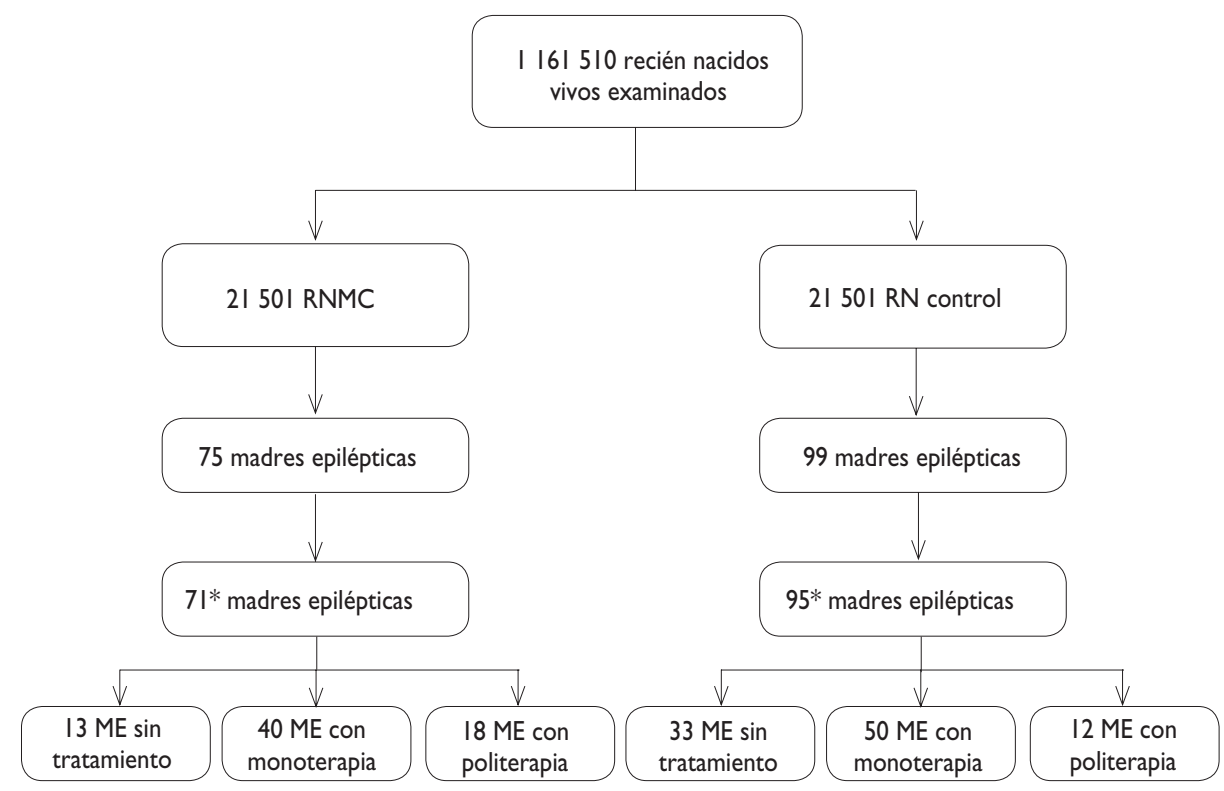

Se excluyeron cuatro casos de cada grupo por falta de información

RNMC: recién nacidos con malformaciones congénitas; RN: recién nacido

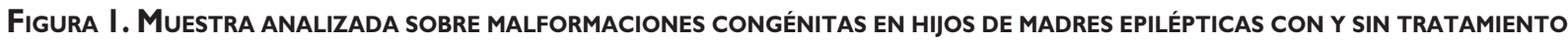
con anticonvulsivantes. MéXico, RYVEMCE I978-20I0. 
De los 166 registros de madres epilépticas restantes, $71(42.8 \%)$ tuvieron un recién nacido con una o más MC mayores y 95 (57.2\%) un producto sin MC. Al agrupar a las madres por tipo de terapia anticonvulsivante recibida, se observaron 90 casos (54.2\%) con monoterapia, 30 casos $(18.1 \%)$ con politerapia y $46(27.7 \%)$ que negaron tratamiento con dichos fármacos. Se observaron 40 RNMC (44.4\%) en el grupo de mujeres con monoterapia, 18 RNMC (60.0\%) en el grupo con politerapia y 13 RNMC (28.3\%) en el grupo de gestantes no tratadas. La frecuencia de RNMC en el grupo de mujeres epilépticas tratadas $(48.3 \%, 58 / 120)$, fue significativamente mayor que en las no tratadas (28.3\% 13/46), ( $R M=2.37$, IC $95 \%$ $1.08-5.40) ; p=0.03$. No se observó diferencia estadísticamente significativa entre la frecuencia de RNMC en el grupo que recibió monoterapia (40/90) con el que recibió politerapia $(18 / 30)$.

En el cuadro I se muestra el tipo de terapia anticonvulsivante empleada y los fármacos más comúnmente prescritos. La difenilhidantoína fue el más frecuentemente utilizado tanto en el grupo de HME con MC como en el grupo de HME sin MC. En el grupo de mujeres que requirieron politerapia, las combinaciones más frecuentes fueron difenilhidantoína+carbamazepina y difenilhidantoína+fenobarbital. Se observaron otras siete diferentes combinaciones en 15 mujeres epilépticas, pero sólo en aquellas cinco que utilizaron tres anticonvulsivantes todos los productos fueron RNMC, en cambio, en las 10 madres restantes que recibieron tratamiento con combinaciones de dos fármacos sólo $50 \%$ de los RN presentó una o más MC.
Del total de 245 hijos que tuvieron las 120 madres epilépticas que utilizaron anticonvulsivantes, 58 (23.7\%) fueron RNMC, 195 fueron hijos de gestantes tratadas con monoterapia, de éstos, 40 (20.5\%) fueron RNMC. De los $50 \mathrm{HME}$ tratadas con politerapia $18(36.0 \%)$ fueron RNMC. Del total de 104 hijos que tuvieron las 46 madres epilépticas no tratadas, 13 (12.5\%) fueron RNMC. La comparación de las frecuencias de HME con MC observadas entre los tres últimos grupos mencionados difirieron significativamente $\left(X^{2} 11.51 ; p<0.01\right)$. Al considerar en conjunto el total de 71 RNMC que tuvieron las 166 mujeres epilépticas y el total de 349 hijos en la descendencia de las mismas, la frecuencia de RNMC fue de $20.3 \%$.

De los 71 RNMC, 19 presentaron más de una MC, siendo ésta un defecto mayor o menor al haberse diagnosticado $106 \mathrm{MC}$ en el total de RNMC. En el cuadro II se muestra el tipo de MC observadas, agrupadas por sistema o región afectada y estratificadas por el tipo de tratamiento utilizado. Si bien no se observaron diferencias significativas entre tratamientos, las $\mathrm{MC}$ de ciertos sistemas o regiones, como las craneofaciales, de piel y del sistema nervioso central, ocurrieron más frecuentemente.

Ciertas MC como microcefalia, anomalías en reducción de miembros y espina bífida mostraron diferencias estadísticas significativas cuando se compararon sus respectivos valores esperados, estimados sobre el total de 21501 RNMC registrados en el RYVEMCE, con los respectivos valores observados en los 71 RNMC presentes en la muestra de madres epilépticas estudiada (cuadro III).

Cuadro I

FreCUENCIA DE RECIÉN NACIDOS CON MALFORMACIONES CONGÉNITAS EN MADRES EPILÉPTICAS TRATADAS CON DIFERENTES ANTICONVUlsivantes dURANTE El PRIMER tRIMESTRE DE LA GESTACIÓN. RYVEMCE, MÉXICO, I978-20I0

Tratamiento

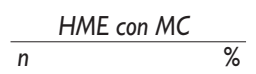

$\frac{H M E \sin M C}{n} \%$

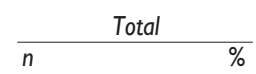

Monoterapia

\begin{tabular}{lcccccc} 
Difenilhidantoína & 19 & 44.2 & 24 & 55.8 & 43 & 100 \\
\hline Carbamazepina & 12 & 37.5 & 20 & 62.5 & 32 & 100 \\
\hline Ácido valproico & 4 & 50.0 & 4 & 50.0 & 8 & 100 \\
\hline Otros & 5 & 71.4 & 2 & 28.6 & 7 & 100 \\
\hline Total de monoterapia & 40 & 44.4 & 50 & 55.6 & 90 & 100
\end{tabular}

Politerapia

\begin{tabular}{lccccccc} 
Difenilhidantoína y carbamazepina & 6 & 54.5 & 5 & 45.5 & 11 & 100 \\
\hline Difenilhidantoína y fenobarbital & 2 & 50.0 & 2 & 50.0 & 4 & 100 \\
\hline Otras combinaciones & 10 & 66.7 & 5 & 33.3 & 15 & 100 \\
\hline Total de politerapia & 18 & 60.0 & 12 & 40.0 & 30 & 100
\end{tabular}

HME: hijos de madre epiléptica, MC: malformaciones congénitas 
Cuadro II

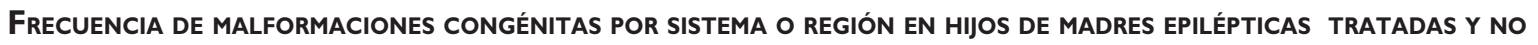
tRatadas. RYVEMCE, MÉXICO, 1978-2010

\begin{tabular}{|c|c|c|c|c|c|c|c|c|}
\hline \multirow[t]{2}{*}{ Sistema o región } & \multicolumn{2}{|c|}{ Sin tratamiento } & \multicolumn{2}{|c|}{ Monoterapia } & \multicolumn{2}{|c|}{ Politerapia } & \multicolumn{2}{|c|}{ Total } \\
\hline & No. & $\%$ & No. & $\%$ & No. & $\%$ & No. & $\%$ \\
\hline SNC & 2 & 10.5 & 10 & 52.6 & 7 & 36.8 & 19 & 100 \\
\hline Cráneo-facial & 8 & 25.8 & 13 & 41.9 & 10 & 32.3 & 31 & 100 \\
\hline Gastrointestinal & 0 & 0.0 & 0 & 0.0 & 3 & 100.0 & 3 & 100 \\
\hline Genitourinario & I & 25.0 & I & 25.0 & 2 & 50.0 & 4 & 100 \\
\hline Cardiovascular & 0 & 0.0 & I & 33.3 & 2 & 66.7 & 3 & 100 \\
\hline Extremidades & 4 & 30.8 & 7 & 53.8 & 2 & 15.4 & 13 & 100 \\
\hline Pared abdominal & 1 & 25.0 & 2 & 50.0 & 1 & 25.0 & 4 & 100 \\
\hline Piel y faneras & 5 & 19.2 & II & 42.3 & 10 & 38.5 & 26 & 100 \\
\hline Otras & 0 & 0.0 & 2 & 66.7 & I & 33.3 & 3 & 100 \\
\hline Total de MC & 21 & 19.8 & 47 & 44.3 & 38 & 35.8 & 106 & 100 \\
\hline
\end{tabular}

SNC: sistema nervioso central, MC: malformaciones congénitas

De los 43 neonatos HME que utilizaron monoterapia con difenilhidantoína, dos presentaron el síndrome embriofetal por hidantoína del recién nacido (perímetro cefálico pequeño, hipoplasia huesos nasales, hipoplasia ungueal e hipertricosis). Una de las 32 madres que recibió tratamiento con carbamazepina tuvo un hijo con espina bífida, hipoplasia nasal, micrognatia e hipertricosis. De las 11 madres que utilizaron politerapia con difenihidantoína + carbamazepina, dos tuvieron un hijo con hipoplasia ungueal y agenesia/ hipoplasia nasal, y otra un RN con anencefalia y paladar hendido. De los cinco hijos de madres que recibieron tratamiento con tres antiepilépticos, uno presentó anomalía en reducción de miembros, hipoplasia ungueal e hipoplasia nasal, y otro, microcefalia, hipoplasia ungueal e hipertricosis, lo que corresponde en ambos casos a la combinación de ácido valproico, difenilhidantoína y carbamazepina.

La frecuencia de parto pretérmino fue significativamente mayor en madres epilépticas, independientemente de no haber recibido tratamiento $26.1 \%$ (12/46), de ser tratadas con monoterapia $25.6 \%$ (23/90) o tratadas con politerapia $23.3 \%$ (7/30) que en madres control $8.4 \%$ (14/166). Las comparaciones de los tres grupos de madres epilépticas con las madres control mostraron los siguientes datos: $(R M=3.83$ IC $95 \%$ 1.47-9.73), $p<0.01$; $(R M=3.73$ IC $95 \%$ 1.71-8.31), $p<0.01$; $(R M=3.30$ IC $95 \%$ $1.01-9.85), p<0.05$, respectivamente. No se observaron diferencias significativas cuando se compararon los tres grupos de madres epilépticas entre sí.

Bajo peso al nacimiento ( $<2500 \mathrm{gr}$ ) en gestaciones de término se observó más frecuentemente en HME con

\section{Cuadro III}

RESULTADO DE LAS COMPARACIONES ENTRE LOS VALORES OBSERVADOS $Y$ ESPERADOS PARA DETERMINADAS MALFORMACIONES CONGÉNITAS EN HIJOS DE MADRES EPILÉPTICAS. RYVEMCE, MÉXICO, 1978-20I0

\begin{tabular}{lcccc} 
Malformación & $\begin{array}{c}\text { Frecuencia } \\
\text { observada }\end{array}$ & $\begin{array}{c}\text { Frecuencia } \\
\text { esperada }\end{array}$ & $\begin{array}{c}\text { Razón } \\
\text { O/E }\end{array}$ & $p^{*}$ \\
Microcefalia & 5 & 0.70 & 7.14 & $<0.01$ \\
\hline Anoftalmia/microftalmia & 2 & $0.6 \mathrm{I}$ & 3.28 & 0.12 \\
\hline ARM & 9 & 2.00 & 4.37 & $<0.01$ \\
\hline Paladar hendido & 3 & 1.04 & 2.88 & 0.09 \\
\hline Onfalocele & 2 & 0.50 & 4.04 & 0.09 \\
\hline Anotia/microtia & 5 & 2.68 & 1.87 & 0.13 \\
\hline Espina bífida & 9 & 4.58 & 1.97 & 0.04 \\
\hline LH+/-PH & 8 & $4.5 \mathrm{I}$ & $\mathrm{I} .77$ & 0.09 \\
\hline Hipospadias & 4 & 1.56 & 2.56 & 0.07 \\
\hline Polidactilia & 3 & 4.48 & 0.67 & 0.35
\end{tabular}

$\mathrm{LH}+/-\mathrm{PH}$ : labio hendido con/sin paladar hendido, ARM: anomalías en reducción de miembros, $\mathrm{P}$ *: Valor de $\mathrm{p}$ para la prueba de distribución de Poisson

MC 6/46, difiriendo significativamente únicamente de los controles (4/152) (prueba exacta de Fisher: $p=0.01$; $(R M=5.55$, IC $95 \%$ 1.24-27.76). No se observaron diferencias significativas entre HME con y sin MC (2/72), y de estos últimos con los controles. Otras variables antropométricas como talla y perímetro cefálico no 
mostraron diferencias significativas entre los grupos analizados entre sí y con el grupo control.

Parto por cesárea solamente mostró una frecuencia significativamente mayor en madres epilépticas con RNMC (54.9\%) que madres control (37.9\%) $(R M=1.97$; $1.09-3.61 ; p<0.05)$, y una frecuencia similar en madres que tuvieron hijos sin $\mathrm{MC}$ y madres control.

Otras variables estudiadas como edad materna y paterna, escolaridad de los progenitores, abortos espontáneos y nivel socioeconómico no mostraron diferencias estadísticas significativas entre los grupos de madres epilépticas con RNMC, madres epilépticas con hijos sin malformaciones y madres control.

\section{Discusión}

La prevalencia de epilepsia en mujeres en edad reproductiva en nuestra muestra fue de $1 / 247$, similar a la ya reportada en otros estudios. ${ }^{5}$ De las madres epilépticas, $72.3 \%$ ingirió uno o más anticonvulsivantes durante el primer trimestre del embarazo, por lo que se observa un riesgo $137 \%$ mayor $(R M=2.37$; IC $95 \% 1.08-5.40 ; p=0.03)$ de tener un RNMC en aquellas madres epilépticas que emplearon uno o más anticonvulsivantes que en las que no recibieron tratamiento.

De las madres epilépticas tratadas, $75 \%$ empleó monoterapia y $25 \%$ politerapia, resultado semejante al observado en una cohorte de madres epilépticas de la población mexicana en la cual la frecuencia de monoterapia fue de $67.1 \%$ y $32.9 \%$ de politerapia. ${ }^{25}$ Otro estudio observacional realizado en el Reino Unido señala cifras cercanas a nuestros resultados para el uso de monoterapia (66.8\%) pero difiere en cuanto al empleo de politerapia (16.6\%). ${ }^{23}$ Las diferencias pudieran deberse, en parte, al tamaño de las muestras estudiadas y también por los criterios en nuestro país sobre las guías de tratamiento y seguimiento de los pacientes epilépticos. La Normal Oficial Mexicana NOM-034-SSA2-20021 para la prevención y control de los defectos al nacimiento señala en el numeral 6.5: "En el primer nivel de atención se llevará a cabo el manejo preventivo de los siguientes factores o condiciones de riesgo generales, en mujeres y parejas en edad fértil (antes, durante y después del embarazo), para prevenir los defectos al nacimiento: 6.5.12. Exposición aguda o crónica a medicamentos (anticonvulsivos, anticoagulantes y ototóxicos)", y en el numeral 6.6 donde se menciona: "En el primer nivel de atención se deben establecer medidas educativas y de promoción de la salud, para disminuir riesgos perinatales y actuar de manera acorde en el caso de mujeres con: 6.6.5 Convulsiones". ${ }^{26}$ Sin embargo, no precisa el tratamiento recomendado en las mujeres epilépticas en edad reproductiva, ni los fármacos que deben evitarse por el potencial teratogénico ya comprobado (ácido valproico, primidona), ni tampoco hace referencia a la recomendación de suplementación con ácido fólico a mujeres epilépticas en edad fértil. ${ }^{27}$

Un estudio reciente ${ }^{23}$ muestra que los anticonvulsivantes más empleados por las mujeres epilépticas que emplean monoterapia son la carbamazepina $(26.7 \%)$, el ácido valproico $(20.6 \%)$ y la lamotrigina (14.4\%). En la presente muestra los anticonvulsivantes más utilizados fueron difenilhidantoína (47.7\%), carbamazepina (33.9\%) y el ácido valproico (9.2\%). La diferencia en el uso de lamotrigina pudiera atribuirse, por una parte, al tipo de medicamentos disponibles en el sector salud y, por otra, al costo elevado de este medicamento.

Llama la atención que el ácido valproico se empleó con menor frecuencia $(9.2 \%)$ en nuestra muestra, muy probablemente debido al importante efecto teratogénico atribuido a este compuesto..$^{27} \mathrm{El}$ empleo de ácido valproico se ha asociado con un mayor riesgo de MC en HME comparado con el empleo de otros anticonvulsivantes y de una mayor frecuencia de alteraciones cognitivas en niños seguidos hasta la edad de tres años. ${ }^{27}$ El riesgo absoluto de tener un niño con espina bífida en el caso de madres expuestas a ácido valproico como monoterapia durante el primer trimestre es de 6.0, 0.5\% para defecto del tabique auricular, $0.3 \%$ para paladar hendido, $0.7 \%$ para hipospadias, $0.2 \%$ para polidactilia y $0.1 \%$ para craniosinostosis. ${ }^{8}$ Todos estos riesgos exceden significativamente lo comúnmente observado en la población general.

La frecuencia de RNMC de mujeres tratadas con politerapia fue significativamente mayor que las tratadas con monoterapia y aún mayor que las no tratadas, lo que concuerda con lo reportado por otros autores. ${ }^{7,18,25}$

Dentro de la variedad de MC diagnosticadas, la microcefalia, las anomalías en reducción de miembros y la espina bífida tuvieron una prevalencia observada significativamente mayor a la esperada. Otras, LHc/ sPH, $\mathrm{PH}$, onfalocele e hipospadias, aunque no alcanzaron diferencias significativas, mostraron una tendencia similar (cuadro III). No hemos encontrado reportes en la literatura médica de casos que presentaran anoftalmia/ microftalmia, anotia/microtia y onfalocele. ${ }^{23,28-30}$

Nuestros resultados no sólo confirman hallazgos previos $^{6}$ que reportan una mayor frecuencia de parto pretérmino en HME sino que ésta fue mayor aún (25.3\%) y una frecuencia similar respecto a bajo peso al nacimiento $(6.78 \%)$.

En contraste con reportes previos, no observamos diferencia entre casos y controles al comparar otras características y antecedentes de los progenitores 
como nivel socioeconómico y antecedente de abortos previos. ${ }^{5,6,14}$

Nuestros resultados corroboran el efecto teratogénico de los anticonvulsivantes utilizados en la muestra estudiada. Además, fue posible identificar algunas MC específicas no reportadas previamente como anoftalmia, microftalmia, anotia, microtia y onfalocele. También se observó un mayor riesgo de RNMC en HME que emplearon anticonvulsivantes congruente con un gradiente teratogénico, pues la frecuencia de RNMC en madres epilépticas no tratadas fue de $12.5 \%$, en tratadas con monoterapia de 20.5 y $36.0 \%$ en las tratadas con politerapia al igual que lo reportado por otros autores. ${ }^{7,18}$

Llama la atención la mayor proporción de $\mathrm{RN}$ con una o más MC mayores en los hijos de mujeres no tratadas con anticonvulsivantes durante el primer trimestre de la gestación (13/46), por lo que este hallazgo sugiere que la epilepsia materna per se favorece una mayor ocurrencia de MC en la descendencia, hecho no confirmado por estudios previos. ${ }^{17}$

Un resultado difícil de explicar es la considerable mayor prevalencia al nacimiento de productos $\mathrm{HME}$ con $\mathrm{MC}$, tanto tratadas con mono o politerapia como no tratadas, en comparación con estudios previos. ${ }^{6-8,12-15,17-19,29,30}$ Por el tipo de MC mayores observadas, un diagnóstico equivocado o un sobre diagnóstico es difícil de aceptar dada la metodología de averiguación en el RYVEMCE. A pesar de que la frecuencia de madres epilépticas en el total de RNMC y sus respectivos controles fue muy similar (1/247) a la reportada en la literatura, ${ }^{4,5}$ quizá las diferencias mencionadas podrían en parte atribuirse a sesgo del entrevistador, el cual, ante una madre con un RNMC, realizará un interrogatorio más acucioso de antecedentes de exposición durante el embarazo tanto en relación con tratamientos como con enfermedades maternas agudas y crónicas. Una forma de confirmar los hallazgos de esta investigación sería la creación de una cohorte de mujeres epilépticas en edad reproductiva de las cuales se registren ciertas variables como inicio de la enfermedad, anticonvulsivantes utilizados, dosis de los mismos y resultado de la gestación. Una alternativa podría ser un estudio multicéntrico dentro del Programa Prioritario de Epilepsia del Sector Salud.

En síntesis, el presente estudio reitera la importancia de un adecuado control médico en las mujeres epilépticas en edad reproductiva sobre la planificación del embarazo, el uso del antiepiléptico con un menor efecto teratogénico y la necesidad de un adecuado asesoramiento genético en este grupo de madres con un mayor riesgo de ocurrencia de malformaciones congénitas en la descendencia.

\section{Agradecimientos}

A todos los médicos pediatras, neonatólogos y genetistas que participaron y participan del programa RYVEMCE que dio origen al presente estudio.

Declaración de conflicto de intereses. Los autores declararon no tener conflicto de intereses.

\section{Referencias}

I. Organización Mundial de la Salud. Epilepsia, nota descriptiva No. 999 [monografía en internet]. Estados Unidos de América: Oficina Regional de la OMS para las Américas (AMRO), 2012 [Consultado 2012 enero I4]. Disponible en: http://www.who.int/mediacentre/factsheets/fs999/es/ index.html

2. Hauser WA, Annegers JF, Rocca WA. Descriptive epidemiology of epilepsy: contributions of population-based studies from Rochester, Minnesota. Mayo Clin Proc 1996;71:576-586.

3. Kalter H. Teratology in the 20th century: environmental causes of congenital malformations in humans and how they were established. Neurotoxicol Teratol 2003;25: 131-282.

4. Quet F, Preux PM, Huerta M, Ramirez R, Abad T, Fragoso G, et al. Determining the burden of neurological disorders in populations living in tropical areas: who would be questioned? Lessons from a Mexican rural community. Neuroepidemiology 201 I;36:194-203.

5. Hiilesmaa VK, Teram K, Bardy AH. Social class, complications, and perinatal deaths in pregnancies of epileptic women: preliminary results of the prospective Helsinki study. En: Janz D et al, eds. Epilepsy, Pregnancy and the child. New York: Raven Press, 1982;3-I4.

6. Yerby MS, Kaplan P, Tran T. Risks and management of pregnancy in women with epilepsy. Cleve Clin J Med 2004; 71 suppl 2:S25-S37.

7. Morrow J, Russell A, Guthrie E, Parsons L, Robertson I, Waddell R, et al. Malformation risks of antiepileptic drugs in pregnancy: a prospective study from the UK Epilepsy and Pregnancy Register. J Neurol Neurosurg Psychiatry 2006;77:193-198.

8. Jentink J, Loane MA, Dolk H, Barisic I, Garne E, Morris JK, et al. Valproic acid monotherapy in pregnancy and major congenital malformations. N Engl J Med 2010;362:2185-2193.

9. Bodendorfer TW. Fetal effects of anticonvulsant drugs and seizure disorders. Drug Intell Clin Pharm 1978;12:1421.

I0. Janz D, Fuchs U. Are anti-epileptic drugs harmful during pregnancy? Dtsch Med Wochenschr 1964;89:241-248.

II. Meadow SR. Congenital abnormalities and anticonvulsant drugs. Proc R Soc Med 1970;63:48-49.

12. Rosa FW. Spina bifida in infants of women treated with carbamazepine during pregnancy. N Engl J Med 199|;324:674-677.

13. Tomson T, Battino D. Teratogenic effects of antiepileptic drugs. Seizure 2008; I7:166-I7I.

14. Swartjes JM, van Geijn HP. Pregnancy and epilepsy. Eur J Obstet Gynecol Reprod Biol 1998;79:3-II.

15. Artama M, Ritvanen A, Gissler M, Isojärvi J, Auvinen A. Congenital structural anomalies in offspring of women with epilepsy-a populationbased cohort study in Finland. Int J Epidemiol 2006;35:280-287.

16. Mutchinick O, Lisker R, Babinski V. The Mexican program of Registration and Epidemiologic Surveillance of External Congenital Malformations. Salud Publica Mex 1988;30:88-100. 
17. Fried S, Kozer E, Nulman I, Einarson TR, Koren G. Malformation rates in children of women with untreated epilepsy: a meta-analysis. Drug Saf 2004;27:197-202.

18. Meador K, Reynolds MW, Crean S, Fahrbach K, Probst C. Pregnancy outcomes in women with epilepsy: a systematic review and meta-analysis of published pregnancy registries and cohorts. Epilepsy Res 2008;8I:I-I3. 19. Hernández-Díaz S, Werler MM, Walker AM, Mitchell AA. Neural tube defects in relation to use of folic acid antagonists during pregnancy. Am J Epidemiol 200I;I53:96I-968.

20. Van Gelder MM, Van Rooij IA, Miller RK, Zielhuis GA, de Jong-van den Berg LT, Roeleveld N. Teratogenic mechanisms of medical drugs. Hum Reprod Update 2010;16:378-394.

2I. Yerby M, Koepsell T, Daling J. Pregnancy complications and outcomes in a cohort of women with epilepsy. Epilepsia 1985;26:631-635.

22. Borthen I, Eide MG, Veiby G, Daltveit AK, Gilhus NE. Complications during pregnancy in women with epilepsy: population-based cohort study. BJOG 2009; I 16:1736-1742.

23. Mawer G, Briggs M, Baker GA, Bromley R, Coyle H, Eatock J, et al. Pregnancy with epilepsy: obstetric and neonatal outcome of a controlled study. Seizure 2010;19:1 12-119.

24. Moore SJ, Turnpenny P, Quinn A, Glover S, Lloyd DJ, Montgomery T, et al. A clinical study of 57 children with fetal anticonvulsant syndromes. J Med Genet 2000;37:489-497.
25. Juárez-Olguín H, Belmont-Gómez A, Flores-Pérez J, BarrancoGarduño LM, Flores-Pérez C. Malformations in newborns associated to anticonvulsant consumption during pregnancy, experience in third level hospital of Mexico. Rev Invest Clin 2008;60:15-20.

26. NORMA Oficial Mexicana NOM-034-SSA2-2002 Para la prevención y control de los defectos al nacimiento [Monografía en internet]. Estados Unidos Mexicanos: Secretaría de Salud, publicada el 27 de octubre de 2003 [Consultado 2012 enero 17]. Disponible en: http://www.salud.gob. $\mathrm{mx} /$ unidades/cdi/nom/034ssa202.html

27. Harden CL, Meador KJ, Pennell PB, Hauser WA, Gronseth GS, French JA, et al. Management issues for women with epilepsy-Focus on pregnancy (an evidence-based review): II. Teratogenesis and perinatal outcomes: Report of the Quality Standards Subcommittee and Therapeutics and Technology Subcommittee of the American Academy of Neurology and the American Epilepsy Society. Epilepsia 2009;50: I237-I246. 28. Bertollini R, Mastroiacovo P, Segni G. Maternal epilepsy and birth defects: a case-control study in the Italian Multicentric Registry of Birth Defects (IPIMC). Eur J Epidemiol; I:67-72.

29. Holmes LB, Harvey EA, Coull BA, Huntington KB, Khoshbin S, Hayes AM, et al. The teratogenicity of anticonvulsant drugs. $N$ Engl J Med 2001;344:1 I32-1 I 38.

30. Obican S, Scialli AR. Teratogenic exposures. Am J Med Genet C Semin Med Genet 20II;157:150-169. 